positive. Symptoms were night sweats only. Blood cultures remained sterile.

Patients 1 and 2 were treated with $200 \mathrm{mg}$ doxycycline daily for 6 weeks with intra-muscular netromycin (150 $\mathrm{mg} /$ day) for 2 weeks. Patient 3 was treated with trimethoprim $160 \mathrm{mg}$-sulfamethoxazole $800 \mathrm{mg}$ tablets twice daily for 10 days because of her pregnancy. Therapeutic abortion was done after 9 days of treatment because of the risk of vertical transmission. Treatment with rifampicin $600 \mathrm{mg} / \mathrm{day}$ and doxycycline $200 \mathrm{mg} /$ day was started. Symptoms disappeared after 4 weeks. Placental cultures were negative.

This laboratory epidemic confirms the professional risk involved in sniffing cultures, a habit common in French laboratories to guide bacterial identification. Delays in seroconversion for two patients indicate that serum surveillance must be for at least 3 months. The index case is atypical, which shows that systematic precautions must be taken whatever the origin of the samples. For patient 3, the mother-to-fetus transmission is unknown, and a large number of cases would have to be followed to allow a conclusion. ${ }^{3}$

*M Grammont-Cupillard, L Berthet-Badetti, *P Dellamonica * Service de Maladies infectieuses et Troptcales, Hôpttal de l'Archet. BP 7906202 Nice Cedex, France

1 Al-Aska AK, Chagla AH. Laboratory-acquired brucellosis. $\mathcal{f}$ Hosp Infect $1989 ; 14: 69-71$.

2 Staszkiewicz J, Lewis CM, Colville J, Zervos $M$, Band J. Outbreak of Brucella melitensis among microbiology workers in a community hospital. F Clin Microbiol 1991; 29: 287-90.

3 Seoud M, Saade G., Awar G, Uwaydah M. Brucellosis in pregnancy. fReprod Med 1991; 36: 441-45.

\section{Oral anticoagulants and fetal loss}

SIR-The EPCOT study group (Oct 5, p 913) ${ }^{1}$ show a relation between fetal loss and inherited thrombophilic defects. The mechanism of impaired placental development and function would certainly be a logical mechanism for the increased fetal loss recorded. However, another factor in fetal outcome was not addressed-namely, the effect of anticoagulation during pregnancy. The investigators do not mention whether any patients received anticoagulants during pregnancy, and if they did, which anticoagulant was used? Since the index group contained individuals with symptoms it would be presumed that some would have received such anticoagulation, and that the control group of healthy individuals would not.

Oral anticoagulants seem to increase fetal and neonatal deaths, ${ }^{2}$ whereas heparin may not have such an effect. In a retrospective study such as EPCOT, oral anticoagulants may have been used in the past, despite reluctance to use them now. Furthermore, the individuals with the highest fetal loss (combined defects and antithrombin deficiency) in this study are those who are likely to have more pregnancy/nonpregnancy associated thrombotic problems ${ }^{3}$ and therefore be candidates for therapeutic or prophylactic anticoagulation during pregnancy, irrespective of whether the defects were diagnosed after the pregnancies. Finally, the increased risk, if any, is low in patients with the factor V Leiden defect; this is a recently denribed familial thrombophilic defect. Since decisions on anticoagulation during pregnancy take into consideration underlying thrombophilic disorders, this group is less likely to have been considered for prophylactic anticoagulants beforc the description of the underlying defect.

The EPCOT report has raised an important issue about fetal loss and possible therapeutic interventions to reduce this loss. Information about the possible effects of anticoagulants on the results presented would be helpful in the interpretation of the data because one can postulate that oral anticoagulation could either increase or decrease fetal loss.

\section{* S A Brown, P W Collins}

Department of Haematology, University Hospital of Wales, Heath Park, Cardiff CF 4 $4 \times W$, UK

1 Preston FE, Rosendaal FR, Walker ID, et al. Increased fetal loss in women with heritable thrombophilia. Lancet 1996; 348: 913-16.

2 Ginsberg JS, Hirsh J. Anticoagulants during pregnancy. Amu Rev Med 1989; 40: 79-86.

3 Conrad J, Horellou MH, Van Dreden P, Lecompte T, Samama $M$. Thrombosis and pregnancy in congenical deficiencies in AT III, protein C or protein S: study of 78 women. Thromb Haemostas 1990; 63: $319-20$.

\section{Authors' reply}

SIR-Brown and Collins raise the interesting and valid point that the increased fetal losses in women with heritable thrombophilia might be influenced by losses attributable to oral anticoagulant thromboprophylaxis during pregnancy. Our results, which concur with those reported by Sanson et al, ${ }^{1}$ were obtained retrospectively at enrolment into the EPCOT study. Unfortunately we do not have detailed information on the use of oral anticoagulants in the women we reported. In an attempt to address the possible role of oral anticoagulants in the observed increased fetal losses we have reanalysed our data, confining our analysis to those 600 of 966 pregnant women who have never had a venous thrombotic event. Since this group of women are the least likely candidates for anticoagulation this approach should greatly reduce the number who receive thromboprophylaxis during pregnancy. The analysis of this group led to a small decrease in the risk estimate but we still find a $25 \%$ excess risk of fetal loss. We anticipate that the prospective data arising out of the EPCOT cohort will clarify these issues.

\section{*F R Rosendaal, F E Preston}

*Department of Clinical Epidemiology, University Hospital Leiden, PO Box 9600 2300 RC Leiden, Netherlands; and Royal Hallamshure Hospital, Sheffreld, UK

1 Sanson BJ, Friedrich PW, Simioni P, et al. The risk of abortion and stillburth in antithrombin-, protein C-, and protem S-deficient women Thromb Haemostas 1996; 75: 387-88.

SIR-In response to Preston and colleagues' report of the EPCOT study, we record three patients with resistance to activated protein $\mathrm{C}$ (APC) who, after thrombosis, had placental abruption despite thromboprophylaxis.

A 25-year-old woman presented with her first pregnancy at 6 weeks' gestation. She had a sinus thrombosis in 1991, but there was no family history of thrombosis. APC resistance was diagnosed (APC ratio 1.61). She was kept on lowmolecular-weight heparin prophylaxis during pregnancy (3075 anti-factor Xa IU, nadroparin, one subcutaneous injection daily). At 17 weeks, placental abruption occurred and the fetus died; there was no fetal anomaly, and the placenta was coated by blood clots and contained several infarctions.

A 20-year-old woman (gravida 2, para 2) was admitted because of labour at 26 weeks' gestation, and fetal distress was identified. After a previous normal delivery she had thrombosis of the inferior vena cava and of the common iliac veins. Subsequently, APC resistance (APC ratio 1.69) and protein $S$ deficiency $(40 \%)$ was diagnosed. The patient's father also had APC resistance. 6 months after her thrombosis she became pregnant, during which lowmolecular-weight heparin (nadroparin) prophylaxis was 
given (one 6150 anti-factor Xa IU subcutaneous injection daily). Despite emerency caesarcan section for placental abruption, the fetus $(570 \mathrm{~g}, 30 \mathrm{~cm})$ died. No fetal malformation was found. The placenta showed numerous small foci of ischaemic necrosis.

A 27 -year-old woman (gravida 4, para 2) was admitted for uterine bleeding at 31 weeks' gestation. She had had a deep venous thrombosis 6 years earlier after a normal delivery. The patient's sister had a stillbirth at 34 weeks. APC resistance was shown (APC ratio 1.65). The patient was kept on low-molecular-weight heparin prophylaxis (3075 anti-Xa IU, nadroparin, one subcutaneous injection daily). At admission fetal death caused by placental abruption and pregnancy-induced hypertension were found. After the stillbirth subarachnoidal haemorrhage was shown in the baby $(1500 \mathrm{~g}, 44 \mathrm{~cm})$ and the placental surface was coated by blood clots. In all patients the postabortal and postpartum courses were uneventful.

There is evidence that APC resistance in symptom-free women might contribute to midtrimester fetal loss. 2 Our three cases indicate that APC resistance in women after clinical presentation of thrombosis comprises fetal outcome more severely than in symptom-free conditions because, despite thromboprophylaxis, the resistance may result in placental abruption, and fetal death. Nevertheless, our experiences suggest that the dose of low-molecular-weight heparin recommended for thromboprophylaxis should be increased during the pregnancy of the thrombophilic patient who has had thrombosis; this may reduce the risk to the fetus, just as in those mothers with antiphospholipid antibodies who were given full-dose heparin therapy ${ }^{3}$ after previous fetal loss.

*Attila Pajor, Géza Sas

*2nd Department of Obstetrics and Gynaecology, Semmelweis University Medical School, 2082 Budapest, Hungan': and National Institute for Haematology and Immunology, Budapest

1 Preston FE, Rosendaal FR, Walker ID, et al. Increased fetal loss in women with heritable thrombophilia. Lancet 1996; 348: 913-16.

2 Rai R, Regan L, Hadley E, Dave $M$, Cohen $H$. Second-trimester pregnancy loss is associated with activated protein $\mathrm{C}$ resistance. Br J Haematol 1996; 92: 489-90.

3 Rosove MH, Tabsh $\mathrm{K}$, Wasserstrum N, Howard P, Hahn BH, Kalunian KC. Heparin therapy for pregnant women with lupus anticoagulant or anticardiolipin antibodies. Obstet Gynecol 1990; 75: 630-34.

SIR-Data from the EPCOT study ${ }^{1}$ show an increased risk of fetal loss in women with thrombophilia, especially in those with antithrombin deficiency or combined defects. For carriers of factor $V$ Leiden no evidence of an excess risk of miscarriage was found and data on the risk of stillbirth were inconclusive. However, no separate analysis of women with homozygous or heterozygous factor $V$ Leiden was undertaken.

We have investigated 27 women homozygous for the factor $\mathrm{V}$ Leiden mutations (median age 36 years, range 21-60). Some were enrolled in the EPCOT study. 26 women had had at least one venous thromboembolic event (VTE); all had the factor $V$ Leiden mutation, which was detected during laboratory evaluation, one woman was symptom-free and her factor $V$ Leiden status was discovered by chance. A standardised questionnaire was completed by personal interview at the participating centres with particular emphasis on obstetric history. Miscarriage was defined as fetal loss before and including the 28th week of gestation, and stillbirth was defined as intrauterine death after the 28 th week of gestation. 18 women had been pregnant at least once (median $=2$, range $1-6$ ) and had in total 42 pregnancies. In two of the pregnancies thromboprophylaxis was carried out. Seven $(17 \%)$ pregnancies ended in miscarriage (three) or stillbirth (four), and nine (21\%) in termination. Onc miscarriage and three stillbirths, but none of the terminations, were followed by deep vein thrombosis (DVT); in one woman DVT was combined with pulmonary embolism. In two of 24 successful pregnancies without thromboprophylaxis DVT occurred during pregnancy and one woman had DVT after delivery.

We conclude that the risk of stillbirth in women homozygous for factor V Leiden $(10 \%)$ is greater than that in healthy women, who have a prevalence of stillbirth of about $1 \%{ }^{1.2}$ The risk of miscarriage was not increased. More than half the fetal losses were followed by VTE. The prevalence of VTE during pregnancy and after delivery in pregnancies with successful outcome was moderately increased, but lower than in women with heterozygous deficiency of antithrombin protein $\mathrm{C}$, or protein $\mathrm{S}$, who have a pregnancy-associated frequency of VTE of 15-51\%. ${ }^{3}$ Thromboprophylaxis could be considered during pregnancy in women homozygous for factor $\mathrm{V}$ Leiden to reduce the increased risk of stillbirth and VTE.

* Claudia Rintelen, Christine Mannhalter, Ingrid Pabinger, Inge Scharrer, Sabine Klinke

*Division of Haematology and Blood Coagulation, Medical Department $\mathrm{H}$; and Department of Molecular Biology, University of Vienna, 1090 Vienna, Austria; and Department of Angiology. University of Frankfurt, Germany

1 Preston PE, Rosendaal FR, Walker ID, et al. Increased fetal loss in women with heritable thrombophilia. Lancet 1996; 348: 913-16.

2 Eschler $G$, Heinegger $H$, Krone HA. Stillbirth-an analysis of 354 cases 1966-88. Geburts Frauenheilk 1991; 51: 293-97.

3 Pabinger I, Schneider B, for the Gesellschaft fur Thrombose-und Hämostaseforschung (GTH) Study Group on Natural Inhibitors. Thrombotic risk in hereditary antithrombin III, protein $\mathrm{C}$, or protein $\mathrm{S}$ deficiency. Arterioscler Thromb Vasc Biol 1996; 16: 742-48.

\section{Drought and malaria retreat in the Sahel, West Africa}

SIR-The Sahel is a belt of dry savanna lining the southern border of the Sahara. Since 1970, rainfall has decreased by nearly $30 \%$ in this region with severe droughts in 1972, 1983, and 1991-92. The impact of dryness on malaria, endemic in the Sahelian belt, has been studied in Senegal (the Niayes) and the Niger Republic (Niger Valley, Zinder, Diffa).

The Niayes are marshy areas, located in north-west Senegal. Mean annual rainfall decreased from $684 \mathrm{~mm}$ (1931-60) to $427 \mathrm{~mm}(1980-89)$, and to $259 \mathrm{~mm}$ (1992) (figure). The rainy season shortened from 6 to 4 months. The same was observed in Niger, where the rainfall at Niamey decreased from $640 \mathrm{~mm}(1950-69)$ to $457 \mathrm{~mm}$ (1981-90). The environment has changed drastically under the combined impact of drought and population growth. Numerous trees were cut for domestic use and stayed unreplaced due to the drought. In Niger and Senegal, marshes with emergent vegetation (cattail) were larval habitats of Anopheles funestus, a major malaria vector. Then, these habirats became dry even after the rainy season, although their soil retained some moisture allowing vegetable cultivation. As a consequence of environmental changes this vector almost disappeared, comparing data recorded before 1970 and after 1988. In Senegal, $A n$ fimestus accounted for $66 \%$ of night-landing collections in 1967 with an average of 33 bites per man per night and a sporozoitic index between 1.2 and $3.1 \%$. In 1991 , this mosquito was no longer captured. ${ }^{1}$ In 1995, despite heavy rains, its populations did not recover because larval habitats were not restored. In Niger, An funestus was also one of the main 